\title{
Use of Pirfenidone in Idiopathic Pulmonary Fibrosis: A Case Study
}

\section{Giacomo Sgalla MD, Stefania Cerri MD, PhD, Roberto Tonelli, Elisabetta Cocconcelli, Martina Garuti, Margherita Ori, and Luca Richeldi MD, PhD}

Int J Clin Rev 2012;08:07 doi: 10.5275/ijcr.2012.08.07

Center for Rare Lung Diseases, University Hospital of Modena, Modena, Italy

Idiopathic pulmonary fibrosis (IPF) is a chronic, progressive, and fatal fibrosing interstitial pneumonia of unknown cause, which is limited to the lungs and occurs primarily in older adults. It is characterized by progressive dyspnea and irreversible loss of lung function [1]. The prognosis is uniformly poor despite heterogeneity in disease progression, with an estimated median length of survival of 2-5 years. No treatment has been shown to be effective. This premise represents the rationale for the development of novel drugs targeting the underlying fibroproliferative process, with the aim of limiting disease progression and attenuating the decline in pulmonary function.

Pirfenidone, a pyridone derivative (5-methyl-1-phenyl-2-[1H]pyridone), is an orally bioavailable molecule that has showed various anti-inflammatory and anti-fibrotic properties, including inhibition of fibroblast proliferation in animal models of lung fibrosis and regulation of transforming growth factor- $\beta$ and tumor necrosis factor- $\alpha$-mediated fibroblast activity in vitro [2-4]. Nevertheless, the exact mechanism of action of pirfenidone remains to be fully clarified.

Several studies have evaluated the safety and the efficacy of pirfenidone in patients with IPF. Azuma and colleagues assessed 
the efficacy of pirfenidone (1800 mg/day) in 107 Japanese patients with IPF in a randomized, placebo-controlled Phase II trial, which showed a reduced decline in the mean change in vital capacity in the treatment arm [5]. These findings led to three Phase III studies - one in Japan and two across North America and Europe. The results of a Phase III study conducted by Taniguchi et al. led to the approval of pirfenidone for the treatment of IPF in Japan [6]. On the other hand, the CAPACITY program included two similar international trials (PIPF-004 and PIPF-006) in 110 centers across North America, Australia, and Europe [7]; the data from these trials indicated that pirfenidone reduced the decline in forced vital capacity (FVC) and had a favorable benefit/risk profile, and therefore represents an appropriate treatment option for patients with IPF. The first of these studies, PIPF-004, was a Phase III randomized, placebo-controlled, 72-week trial that had three arms: high-dose pirfenidone (2403 mg/day), low-dose pirfenidone (1197 $\mathrm{mg}$ /day), and placebo. Enrollment proceeded in a 2:1:2 ratio and included a primary outcome of absolute percentage change in predicted FVC between enrollment and week 72 . At week 72, pirfenidone $2403 \mathrm{mg} /$ day significantly reduced the mean decline in percent-predicted FVC, compared with placebo (-8.0\% vs. -12.4 $\%$, respectively), and the proportion of patients with FVC declines of $>10 \%$.

The 72-week Phase III trial PIPF-006 was undertaken in order to expand these promising results, comparing the effect of high-dose pirfenidone to placebo with randomization in a 1:1 ratio. Unfortunately, at the end of the study a beneficial effect on the primary endpoint of decline in percent-predicted FVC was not achieved ( $\mathrm{p}=0.501)$, but further analysis revealed that this was because of a more-robust placebo effect. Altogether, the treatment effect was significant at weeks 12, 24, 36, and 48.

The safety profile of pirfenidone is excellent, and it appears to be generally well tolerated. The most common side effects in the 
Phase II and Phase III trials were gastrointestinal upset, fatigue, nausea, anorexia, and dermatological problems, including photosensitivity. Despite these adverse events, the number of patients who discontinued the drug was low.

Case report

A 77-year-old, non-smoking, Italian female with an allergy to acetylsalicylic acid and who was affected by anxious-depressive syndrome presented with dry cough in June 2006, followed by the onset of exertional dyspnea in October of the same year. Because of worsening of her dyspnea, the patient underwent a chest X-ray that showed a consolidation (compatible with the diagnosis of bronchopneumonia), which was effectively treated with antibiotics and steroids. The following chest X-ray showed clearing of the area of consolidation, but cough and breathlessness persisted.

Therefore, on 28 February 2007 the patient underwent a highresolution computed tomography scan of the chest, which showed evidence in three different basal lung regions of diffuse interstitial lung disease characterized by predominantly bibasal and peripheral reticular opacities, traction bronchiectasis, honeycomb lung destruction, and irregular areas of consolidation with no ground glass opacities (Figure 1). The pattern was considered to be consistent with usual interstitial pneumonia. In order to exclude other known causes of pulmonary fibrosis, a bronchoscopy with bronchoalveolar lavage was performed and did not provide evidence for alternative diagnoses. Based on the patient's clinical history, other secondary causes of interstitial lung disease (such as connective tissue diseases or drug toxicity) were also excluded. Thus, a diagnosis of IPF was finally set. At this point, the patient agreed to enrolment in the PIPF-004 clinical trial. In May 2007, she started treatment with the pirfenidone at the dose of 2403 $\mathrm{mg}$ /day, divided into three tablets three times a day.

In June 2007, after 6 weeks of treatment, the patient reported a reduction in cough symptoms and decreased appetite. Between 
June and September 2007, she showed subjective improvement in respiratory symptoms and a further reduction in cough.

In October 2007, the patient came to our attention for an unscheduled visit because of the onset of general malaise, hypotension, dizziness, and anorexia, with altered perception of smell and taste. As a consequence of these adverse events, most likely due to the patient's intolerance to the highest dosage of the pirfenidone, the dosage was reduced to $1800 \mathrm{mg} /$ day, divided into two tablets three times a day.

Nevertheless, at week 24 of the study, the patient's clinical conditions were further improved, meaning that the reduced drug dosage still had an effect on the patient's symptoms. Sporadic episodes of tracheitis, pharyngitis, bronchitis, and labyrinthitis occurred, but all of these were effectively treated.

During the study, the patient underwent several follow-up visits in which pulmonary function tests with diffusing lung capacity for carbon monoxide were performed, together with blood tests, a 6min walking test, and electrocardiogram. The University of California, San Diego Shortness of Breath questionnaire, the St George's Respiratory Questionnaire and the World Health Organization Quality of Life-100 questionnaire were used at the beginning of the trial to gain information about dyspnea and quality of life. At the end of the PIPF-004 study, the patient's clinical conditions were stable and good, notwithstanding the persistence of bibasal, bilateral, Velcro-type crackles.

In October 2008, the patient was included in the PIPF-012 rollover study. Until March 2012 the patient underwent follow-up visits at our center in Modena, Italy, with quarterly assessment of pulmonary function and blood tests. During nearly 4 years of additional follow-up the patient did not present any other sideeffect related to pirfenidone, nor any acute exacerbations of IPF. 
Nevertheless, other sporadic episodes of pharyngitis and bronchitis occurred, but these were all effectively treated with antibiotics. In March 2012, the PIPF-012 study ended; given the persistent stability of her clinical condition, the patient was admitted to continue receiving pirfenidone under the European Named Patient Program upon approval by the local ethics committee.

Since May 2007, the patient did not present any clinically significant impairment in her lung function parameters, with substantial stability in FVC. Data from pulmonary function tests (one for each year from May 2007 to March 2012) are shown in Table 1. These results are better than that expected from the published results of the CAPACITY trials; nevertheless, it is not known whether this functional stability is related to the efficacy of pirfenidone, as it could also be because of the favorable course of the fibrotic process, or the natural history of the disease for this particular patient. On the other hand, the hypothesis of a drugrelated benefit is supported by the improvement in respiratory symptoms experienced by the patient from a few weeks after starting treatment with pirfenidone, and continuing after the dosage reduction from $2400 \mathrm{mg} /$ day to $1800 \mathrm{mg}$ /day; this means that 1800 $\mathrm{mg} /$ day could be considered as the lowest effective dosage for this patient.

The patient has suffered from some of the most frequent adverse events reported for pirfenidone in the CAPACITY trials, such as general malaise, anorexia, and gastrointestinal symptoms. These effects disappeared with the reduction of the drug dosage from $2400 \mathrm{mg} /$ day to $1800 \mathrm{mg}$ /day, confirming the good tolerability of pirfenidone.

Disclosures: Dr Richeldi participated as a clinical investigator in the CAPACITY trials and received compensation for scientific speaking and consultation from InterMune Inc. Other authors have no financial disclosures. 
Address for correspondence: Luca Richeldi, Centro Malattie Rare del Polmone, Policlinico di Modena, Via del Pozzo 71, Modena 41100, Italy. Email: luca.richeldi@unimore.it.

\section{References}

1. Raghu G, Collard HR, Egan J et al. An official ATS/ERS/JRS/ALAT statement: idiopathic pulmonary fibrosis: evidence-based guidelines for diagnosis and management. Am J Respir Crit Care Med 2011;183:788-824. Reference Link 2. Gadekar SM, inventor. Affiliated Medical Research Inc, assignee. 5-Methyl-1-phenyl-2-(1H)-pyridone compositions and methods of use. US patent 3,974,281. 1976 Aug 10.

3. Margolin SB, inventor. InterMune Inc, assignee. Composition and method for reparation and prevention of fibrotic lesions. US patent 5,310,562. 1994 May 10.

4. Iyer SN, Wild JS, Schiedt MJ et al. Dietary intake of pirfenidone ameliorates bleomycin-induced lung fibrosis in hamsters. $J$ Lab Clin Med 1995;125:779-85.

5. Azuma A, Nukiwa T, Tsuboi E et al. Double-blind, placebocontrolled trial of pirfenidone in patients with idiopathic pulmonary fibrosis. Am J Respir Crit Care Med 2005;171:1040-7. Reference Link

6. Taniguchi H, Ebina M, Kondoh Y et al.; Pirfenidone Clinical Study Group in Japan. Pirfenidone in idiopathic pulmonary fibrosis. Eur Respir J 2010;35:821-9. Reference Link

7. Noble PW, Albera C, Bradford WZ et al. Pirfenidone in patients with idiopathic pulmonary fibrosis (CAPACITY): two randomised trials. Lancet 2011;377:1760-9. Reference Link 


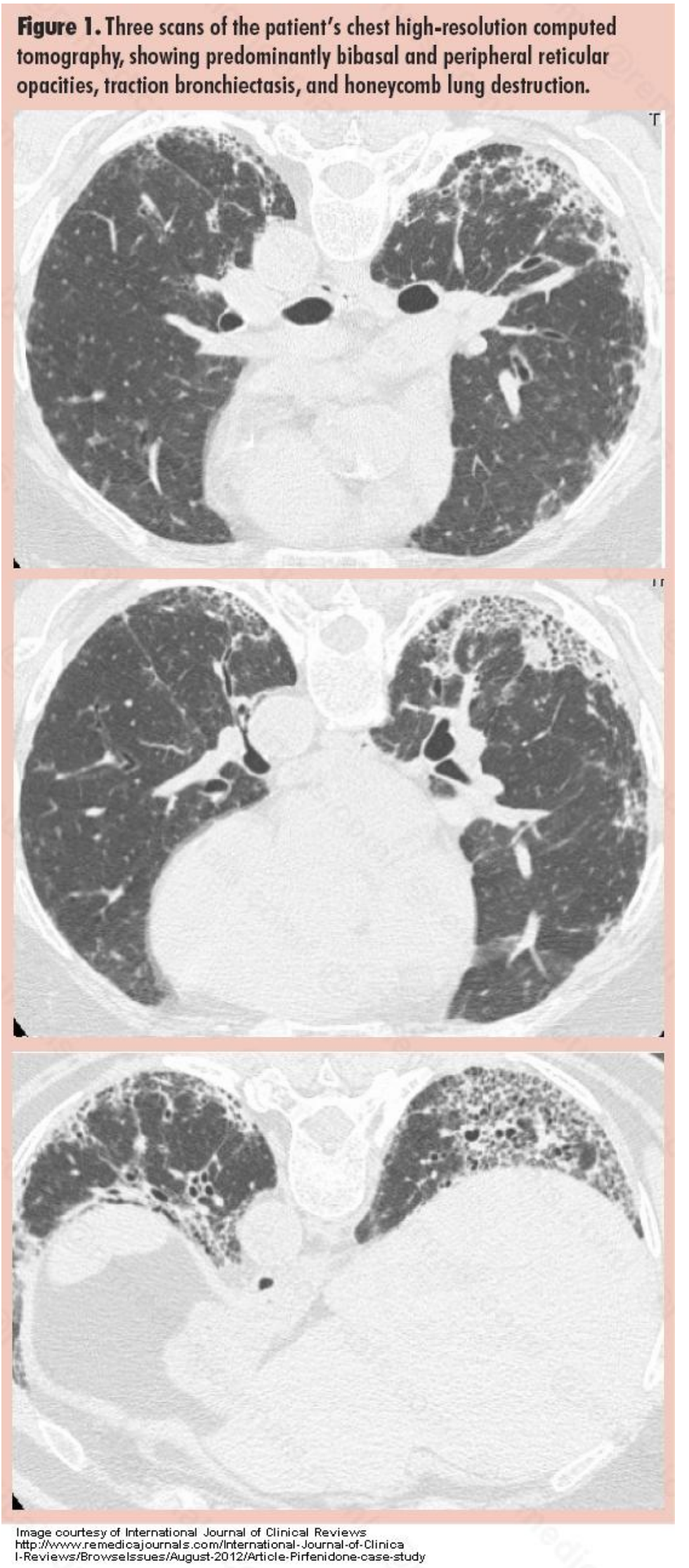




\begin{tabular}{|c|c|c|c|c|c|c|}
\hline Pulmonary function test & 2007 & 2008 & 2009 & 2010 & 2011 & 2012 \\
\hline FVC & 91 & 98 & 113 & 119 & 92 & 95 \\
\hline $\mathrm{FEV}_{1}$ & 90 & 101 & 115 & 113 & 97 & 98 \\
\hline $\mathrm{FEV}_{1} / \mathrm{FVC}$ & 80 & 82.77 & 81.77 & 66.64 & 84.15 & 82.43 \\
\hline TLCO & 54 & 56 & 62 & 56 & 30 & 37 \\
\hline $\mathrm{KCO}$ & 64 & 63 & 58.5 & 53 & 43 & 64 \\
\hline
\end{tabular}

